\title{
Invasive carcinoma no special type, pleomorphic carcinoma: Case report of a rare variant of invasive breast carcinoma
}

\author{
Deepu Mathew Cherian', C. N. Sai Shalini2,", Leena Dennis Joseph³, Bhawna Dev ${ }^{4}$, Vishwanath \\ M. Pai ${ }^{5}$ \\ ${ }^{1}$ PG Student, ${ }^{2,3}$ Associate Professor, Dept. of Pathology, ${ }^{4}$ Professor, Dept. of Radiodiagnosis, Sri Ramachandra \\ Medical College and Research Institute, Chennai, ${ }^{5}$ Professor, Dept. of Surgery
}

\section{*Corresponding Author:}

Email: saishalini_cn14@yahoo.com

\begin{abstract}
Invasive breast carcinoma - No special type (NST), Pleomorphic carcinoma is a rare morphological variant of invasive carcinoma NST. This is a case report of a 30 year old nulliparous woman who presented with a tender palpable mass in her right breast. Microscopic examination of the breast lump was predominantly composed of pleomorphic and bizarre cells. No positive lymph nodes or distant metastasis was present. This case report is to emphasise on the importance of identifying this variant of breast carcinoma because of its prognostic significance.
\end{abstract}

Keywords: Carcinoma breast, Pleomorphic carcinoma, Pleomorphic bizarre tumor cells.

Received: $23^{\text {rd }}$ November, 2017

Accepted: $26^{\text {th }}$ December, 2017

\section{Introduction}

Invasive breast carcinoma - NST, Pleomorphic carcinoma is a rare variant of invasive carcinoma. In 2012 World Health Organization (WHO) classification1, Pleomorphic carcinoma is classified as a rare morphological variant of invasive carcinoma NST. The unique feature of this carcinoma is proliferation of pleomorphic and bizarre tumour cells (comprising of more than fifty percent of the tumour cells). This variant is usually seen in association with adenocarcinoma or adenocarcinoma with spindle cell differentiation. This variant shows a high degree of variation in nuclear size and characteristically has giant tumour cells which are often multinucleated. Literature suggests that pleomorphic carcinoma has an aggressive behaviour and poor clinical outcome. We report a case of Invasive carcinoma NST, Pleomorphic carcinoma of the breast in a 30 year old woman.

\section{Clinical history}

A 30 year old female came with the chief complaint of a painful lump in the right breast for the past 2 months. She had no significant past or family history. She was a nulliparous woman, married for six years. Breast examination revealed a palpable tender lump in the lower outer quadrant of right breast measuring $5 \times 5 \mathrm{~cm}$. Skin over the swelling appeared normal. Examination of left breast was unremarkable. Ultrasonography of the right breast showed an irregular, ill defined, mixed echogenic lesion measuring $6.5 \times 1 \times \quad 4 \quad x \quad 5.3 \quad \mathrm{~cm}$ at $3-4$ o'clock position with few cystic spaces showing internal echoes and septations (Fig 1). The final impression on ultrasound of the breast was BIRADS IV B lesion which indicates moderate suspicion for malignancy. Fine needle aspiration cytology was done from the lesion which showed clusters of benign ductal epithelial cells and myoepithelial cells admixed with cyst macrophages, suggestive of fibrocystic change. Excision biopsy was done and the specimen was sent for histopathological examination.

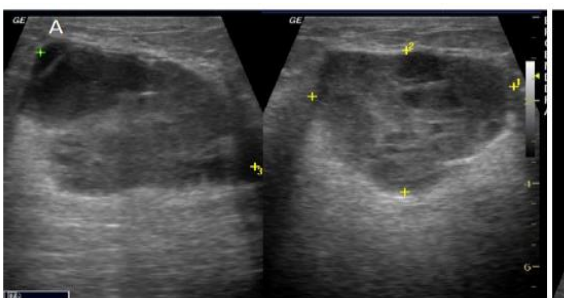

Indian Journal of Pathology and Oncology, January-March, 2018;5(1):157-160

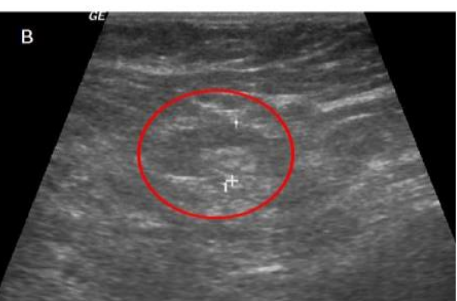

157 
Fig. 1: A: Ultrasonography images showing an irregular, ill defined, mixed echogenic lesion with few cystic spaces with internal echos and septations; B: Lymph node (in red circle) showing preserved fatty hilum

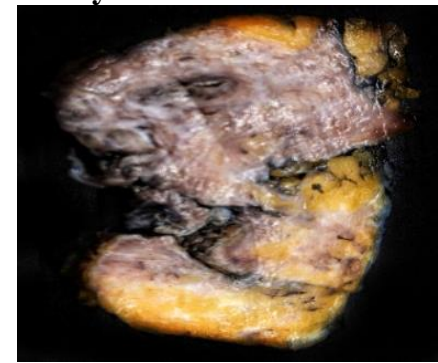

Fig. 2: Gross specimen showing a grey white grey tan lesion measuring $2.2 \times 2 \times 1 \mathrm{~cm}$

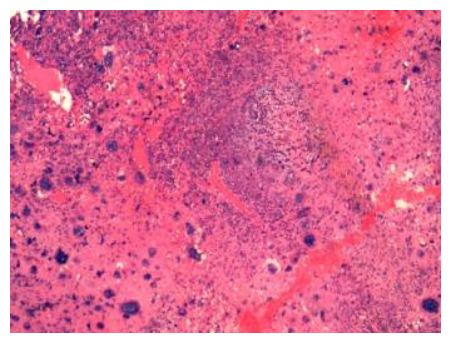

Fig. 3

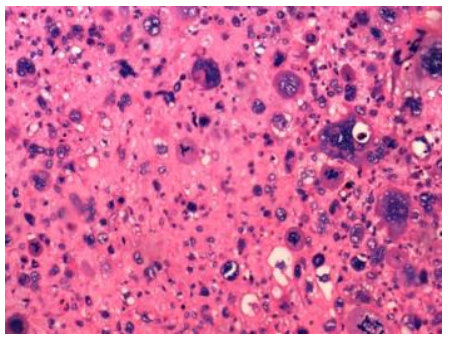

Fig. 4

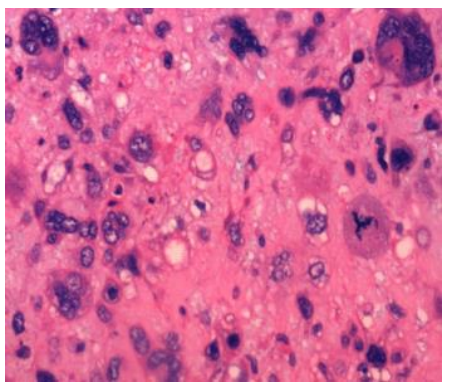

Fig. 5

Fig. 6

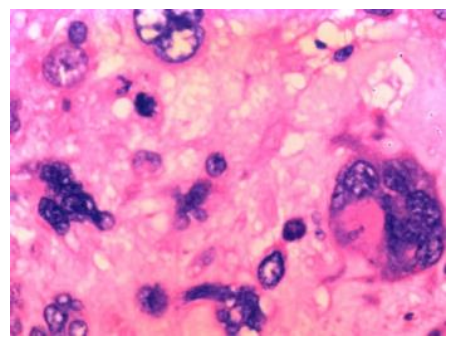

Fig. 3-6: H\&E section at 4x, 10x, 20x and 40x magnification respectively showing pleomorphic and bizarre giant cells

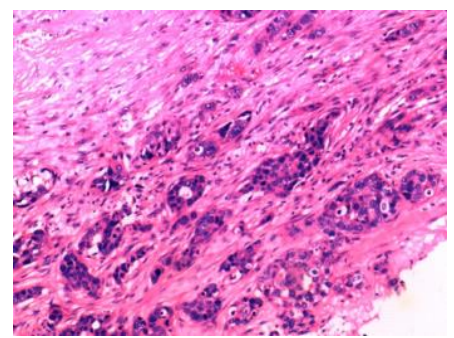

Fig. 7: $H \& E$ section at $10 x$ magnification showing invasive carcinoma NST.

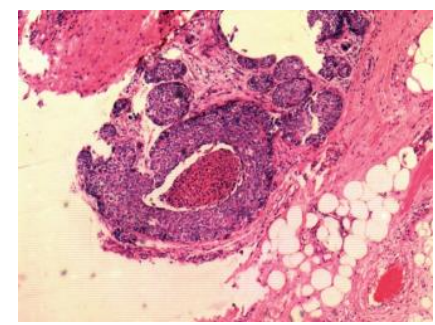

Fig. 8: H\&E section at $4 x$ magnification showing high grade ductal carcinoma in-situ with comedo necrosis

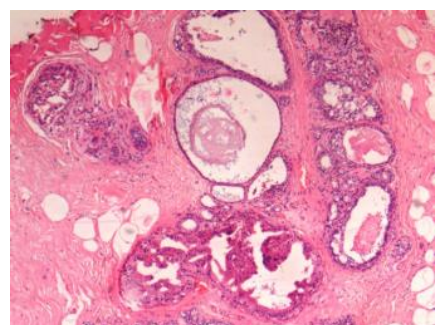

Fig. 9: $H \& E$ section at $4 x$ showing areas of fibrocystic component

Gross: The specimen was multiple grey-white to grey-yellow soft tissue fragments altogether measuring $9 \times 5 \times 4 \mathrm{~cm}$. Cut surface of the largest fragment showed a grey white - grey tan lesion measuring $2.2 \times 2 \times 1 \mathrm{~cm}$ (Fig 2).

Microscopy: The lesion showed predominantly pleomorphic and bizarre giant cells comprising $70 \%$ of the lesion (Fig 3-6) and invasive carcinoma NST comprising rest of the $30 \%$ (Fig. 7). The pleomorphic cells were large having bizarre nuclei. High grade ductal carcinoma in-situ with comedo necrosis was also seen (Fig 8). Mitotic count was 25/10 HPF. With all these features, a final diagnosis of Invasive Carcinoma NST, Pleomorphic Carcinoma was rendered. The adjacent area showed extensive 
areas of fibrocystic component (Fig 9). Subsequently, modified radical mastectomy was done. The specimen measured $24 \times 10.5 \times 4 \mathrm{~cm}$ and weighed 800 grams. Serial sectioning showed tumour measuring $4 \times 3 \times 2.5 \mathrm{~cm}$ in the lower outer quadrant. Microscopy of the lesion from the mastectomy specimen showed a similar histology as that of the excision biopsy specimen. 19 axillary lymph nodes were identified; all of them were free of tumour. Hence the final impression was given as Right modified radical mastectomy pT3N0 Invasive Carcinoma NST - Pleomorphic carcinoma, Nottingham histological grade 3.

\section{Discussion}

Invasive breast carcinoma - NST, Pleomorphic carcinoma is a recent entity which has distinctive and particular morphological features. It is predominantly made up of cells having marked variability in size and shape; most of these cells are large cells with multiple nuclei. This variant of invasive carcinoma was initially described by Silver SA and Tavassoli FA. They concluded that when all the grade III invasive breast carcinomas are taken, this variant occupies the extreme end of the spectrum. ${ }^{2}$ In 2012 WHO classification, this tumour is considered as a variant of invasive carcinoma NST. Pleomorphic carcinoma is seen in an age group ranging from 23 to 96 years with a mean age of 50 years. $^{2,3}$ The most common presentation of pleomorphic carcinoma is a palpable mass with a mean size of $15 \mathrm{~cm}$. Usually these tumours are circumscribed lesions, few showing surface ulceration or central necrosis. On microscopy, $>50 \%$ of the tumour is composed of pleomorphic cells and multinucleated cells. Tumours are usually grade 3 with high mitosis and central necrosis. Adjacent DCIS may be present. Presence of spindle cell component and squamoid differentiation are also reported. ${ }^{2}$ Neoplastic cannibalism is seen in some cases in which tumour cells were engulfed by other tumour cells. ${ }^{3}$ This has been described in pleomorphic carcinomas of other sites like lung. ${ }^{4}$ These tumours are usually negative for ER (estrogren receptor), PR (progesterone receptor) and Her2neu, but few cases have shown overexpression of Her2. ${ }^{5}$ Axillary lymph node metastasis is seen in $50 \%$ cases. ${ }^{1}$ Pleomorphic carcinoma has an overall poor prognosis. If the tumour is showing areas of spindle cells and if the tumour size is more than $5 \mathrm{~cm}$, then it will have a more adverse outcome. ${ }^{3}$
Pleomorphic cells can also be seen in other breast tumours. They are invasive pleomorphic lobular carcinoma, invasive carcinoma NST Carcinoma with osteoclast-like giant cells and carcinoma with choriocarcinomatous features. ${ }^{6}$ Microscopic features and immunohistochemistry markers are needed to arrive at a diagnosis. Pleomorphic lobular carcinoma is a subtype of Invasive lobular carcinoma. The tumour cells have a linear arrangement and they lack E Cadherin expression. ${ }^{7}$ Carcinoma with osteoclast like giant cells have giant cells which shows $C D$ 68 positivity and CK negativity. ${ }^{8}$ Carcinoma with choriocarcinomatous features have pleomorphic cells positive for beta HCG (human chorionic gonadotropin). ${ }^{9}$

Pleomorphic carcinoma of breast is a rare variant of invasive carcinoma NST as described by 2012 WHO classification of breast tumours. It is important to identify this tumour due to prognostic significance especially when associated with a tumour size of more than $5 \mathrm{~cm}$ or a spindle cell component.

\section{References}

1. Ellis IO, Collins L IS and MG. WHO

Classification of Tumours of the Breast. In: Lakhani SR, Ellis IO, Schnitt SJ TP and van de VM, editor. WHO Classification of Tumours of the Breast. Fourth Edi. 2012. p. 34-8.

2. Silver SA, Tavassoli FA. Pleomorphic carcinoma of the breast: clinicopathological analysis of 26 cases of an unusual high-grade phenotype of ductal carcinoma. Histopathology. 2000 Jun 1;36(6):505-14.

3. Nguyen C V., Falcón-Escobedo R, Hunt KK, Nayeemuddin KM, Lester TR, Harrell RK, et al. Pleomorphic Ductal Carcinoma of the Breast: Predictors of Decreased Overall Survival. Am J Surg Pathol. 2010 Apr;34(4):486-93.

4. Ginsberg SS, Buzaid AC, Stern H, Carter D. Giant cell carcinoma of the lung. Cancer. 1992 Aug 1;70(3):606-10.

5. Zhao J, Lang R, Guo X, Chen L, Gu F, Fan Y, et al. Clinicopathologic characteristics of pleomorphic carcinoma of the breast. Virchows Arch. 2010 Jan 16;456(1):31-7.

6. Tang $\mathrm{H}$, Liu $\mathrm{F}$, Li $\mathrm{H}$, Huang $\mathrm{X}$, Zhao $\mathrm{T}$. Pleomorphic carcinoma of breast: a case report and review of literature. Int $\mathbf{J}$ Clin Exp Pathol. 2014;7(8):5215-20.

7. Wahed A, Connelly J, Reese T. E-cadherin expression in pleomorphic lobular carcinoma: An aid to differentiation from ductal carcinoma. Ann Diagn Pathol. 2002 Dec;6(6):349-51.

8. Kurokawa K, Mouri Y, Asano A, Kamei K, Iwata Y, Isogai M, et al. Pleomorphic carcinoma with osteoclastic giant cells of the breast: Immunohistochemical differentiation between coexisting neoplastic and reactive giant cells. Pathol Int. 2009 Feb;59(2):91-7.

9. Horne $\mathrm{CH}$, Reid IN, Milne GD. Prognostic significance of inappropriate production of 
pregnancy proteins by breast cancers. Lancet (London, England). 1976 Aug 7;2(7980):279-82. 